\title{
Organizações Regionais para o Ordenamento Pesqueiro: 0 poder ouve a ciência?!
}

\author{
Regional Fisheries Management Organizations: \\ is power listening to science?
}

DOI: $10.21530 /$ ci.v13n1.2018.689

Leandra Regina Gonçalves²

\section{Resumo}

Um dos desafios à governança global é garantir a eficácia dos regimes internacionais que buscam reduzir ou solucionar problemas. O manejo dos estoques de peixes, como os atuns, é um bom exemplo da magnitude desse desafio. Nesse sentido, foram criadas as organizações regionais para o ordenamento pesqueiro, para resolver a crise internacional de pesca. O papel das comunidades epistêmicas e a forma como se dá sua influência nas decisões políticas são dois dos fatores considerados cruciais para a eficácia dos regimes internacionais. Então, através de uma avaliação do desenho institucional dos painéis científicos da Convenção sobre a Conservação dos Recursos Vivos Marinhos Antárticos, Convenção Internacional para a Conservação dos Atuns e a Convenção para a Conservação do Atum do Sul, essa pesquisa buscou responder se o poder ouviu a ciência e se isso trouxe mais eficácia para o manejo pesqueiro. Concluiu-se que os tomadores de decisão podem ouvir a ciência e isso pode resultar em maior eficácia para o acordo, porém, é necessário que o desenho dos painéis científicos possa propiciar que conhecimentos e reivindicações científicas sejam desenvolvidos de forma isolada da política. Na prática, isso ainda não se concretizou plenamente.

Palavras-chave: Organizações Internacionais; OROPs; Comunidades Epistêmicas; Construtivismo.

1 Esta pesquisa foi realizada graças ao apoio da bolsa CAPES de doutorado, bem como da bolsa CAPES/Fulbright para Doutorado Sanduíche. Também não poderia deixar de mencionar o apoio da bolsa de mobilidade Santander.

2 Doutora em Relações Internacionais pela Universidade de São Paulo; Fulbright Alumni, Young Fellow da Plataforma Brasileira sobre Biodiversidade e Serviços Ecossistêmicos (BPBES/CNPq); Pesquisadora-colaboradora no Núcleo de Estudos e Pesquisas Ambientais (NEPAM/Unicamp).

Artigo submetido em 11/07/2017 e aprovado em 30/12/2017. 


\section{Abstract}

The role of epistemic communities and the path they follow to influence policies are the keys to answering the main question posed here: when and how did power listen to science? One of the greatest challenges for the promotion of contemporary global environmental governance is to ensure the effectiveness of institutions seeking to reduce or solve problems in this new age of the Anthropocene. The management of highly migratory fish stocks, such as tuna, is a good example of the magnitude of this challenge. The Regional Fisheries Management Organizations have emerged to deal with this international fisheries crisis. Through an evaluation of the institutional design of the Scientific Panels on the Convention for the Conservation of Antarctic Marine Living Resources, the International Convention for the Conservation of Tuna and Related Tunas and the Convention for the Conservation of the Southern Tuna, this research concludes that the power may listen to science, and this can result in greater agreement effectiveness. However, it is necessary that scientific panels are designed to promote a better flow of information to decision makers and that knowledge and the scientific claims are developed insulated from politics, and in practice, this has not yet happened.

Keywords: International Organizations; Regional Fisheries Management Organizations; Epistemic Communities; Constructivism.

\section{Introdução}

No debate acadêmico contemporâneo sobre a política mundial, a "governança global" é um dos temas mais relevantes e de intenso debate (DINGWERTH; PATTBERG, 2006). Mais de duas décadas após a publicação do “Governing without Government” (ROSENAU; CZEMPIEL, 1992) e da publicação da Comissão de Governança Global, “Our Global Neighbourhood”, em 1995, o tema evoluiu, se consolidou e ainda desperta grande interesse nos mais diversificados temas, tais como: direitos humanos (RUGGIE, 2014), saúde (YOUDE, 2017), comércio (RODRIK, 2001), segurança (DUFFIELD, 2014), meio ambiente (YOUNG, 1999) entre outros.

Alguns autores entendem governança nos termos de modos não hierárquicos de governo, onde atores não estatais e diversos segmentos da sociedade participam na formulação e implementação de políticas públicas. Outros compartilham uma visão mais abrangente, reconhecendo que diferentes modos de governança (às vezes em combinação e/ou tensionamento) estão presentes em diferentes 
sistemas (modos hierárquicos, colaborativos ou autônomos). Partindo da definição de governança global cunhada por Rosenau (1995, p. 13)3:

A governança global significa mais do que instituições e organizações formais através das quais a gestão de assuntos internacionais é ou não realizada. O sistema das Nações Unidas e os governos nacionais são certamente centrais para a condução da governança global, mas eles são apenas parte do quadro geral (...) a governança global é concebida para incluir sistemas de governo em todos os níveis da atividade humana - da família à organização internacional - em que a busca dos objetivos através do exercício do controle tem repercussões transnacionais.

E adicionando novos atores e interdependência, Biermann (2008) propôs que governança é:

o inter-relacionado e integrado sistema formal e informal de regras e redes de atores em todos os níveis da sociedade humana (do local ao global) que são estabelecidos para influenciar a coevolução entre sistemas humanos e naturais de maneira que assegure o desenvolvimento sustentável da sociedade humana. (BIERMANN, 2008, p. 281)

E foi nesse sentido que se abriu importante área de estudo voltada a discutir a eficácia de acordos internacionais e regimes (STOKKE, 2001; ANDRESEN, 2016), que fazem parte de um sistema de governança e que contribuem para o desenvolvimento do multilateralismo (KARNS; KAREN, 2004; FORMAN; SEGAAR, 2006). Há de se considerar que a temática está longe de estar totalmente consolidada e a análise de quais fatores tornam a cooperação internacional mais ou menos efetiva provavelmente será um dos aspectos mais importantes da agenda de pesquisa em governança global contemporânea (DESOMBRE, 2007; YOUNG, 2017).

A governança ambiental global, por sua vez, é uma das áreas que tem se beneficiado desse debate e contribuído com significativos aportes (YOUNG, 1999; MILES et al., 2002; DESOMBRE, 2007). Por que alguns regimes ambientais internacionais têm mais sucesso do que outros? Por que alguns regimes ambientais têm um impacto maior do que outros? Essas são questões levantadas por

3 "Global governance refers to more than the formal institutions and organizations through which the management of international affairs is or is not sustained. The United Nations system and national governments are surely central to the conduct of global governance, but they are only part of the full picture (...) global governance is conceived to include systems of rule at all levels of human activity - from the family to the international organization - in which the pursuit of goals through the exercise of control has transnational repercussions". 
pesquisadores dedicados a compreender o sistema internacional onde esses acordos atuam, tais como Young $(1999,2017)$ e Miles (2002).

A presente pesquisa, inserida no âmbito da governança ambiental global, busca discutir a eficácia de acordos ambientais, sobretudo abordando a possibilidade do surgimento e da influência de uma comunidade epistêmica nos moldes da definição de Haas (1992), onde um grupo de especialistas é capaz de produzir e divulgar conhecimento científico e, mais do que isso, influenciar a tomada de decisão no cenário internacional trazendo maior eficácia ao acordo. E, nesse sentido, surge a principal pergunta a ser respondida nesse trabalho: o poder ouve a ciência?

Essa abordagem analisa a influência de grupos de especialistas sobre a reformulação de objetivos nacionais e/ou internacionais e indica como Estados e líderes podem perceber que novas atitudes e procedimentos de decisão política são necessários para enfrentar desafios contemporâneos, além de acordos e negociações globais.

Para manter um maior aprendizado social (social learning) dentro do escopo de um acordo, o desenho de um regime deve garantir que a construção do conhecimento científico seja isolado da política e crie procedimentos claros para a conexão da ciência com a tomada de decisão. Tal conexão geralmente é feita pelo que Haas cunhou como o "cinturão de transmissão", formado por um grupo de cientistas amplamente reconhecido como uma "comunidade epistêmica" (HAAS, 1992) ${ }^{4}$.

Buscando o diálogo sobre o papel das comunidades epistêmicas em um contexto de governança, foram selecionados três acordos internacionais de ordenamento pesqueiro, com o objetivo de estabelecer um quadro de análise para examinar o desenho institucional e compreender o papel da ciência no desempenho das negociações e sua possível consequência para a eficácia do acordo.

Assim, será primeiramente apresentado o papel das comunidades epistêmicas e, então, serão analisadas empiricamente três organizações regionais de ordenamento pesqueiro (OROPs) - RFMOs em inglês: CCAMLR, ICCAT e CCSBT, para ilustrar a abordagem.

4 Essa abordagem foi aplicada em diversos tópicos e níveis, desde o local (INOUE, 2003) até o global (CROSS, 2013), merecendo, inclusive, uma edição especial da Revista da Organização Internacional em 1992. 


\section{O papel das comunidades epistêmicas nos regimes internacionais}

Foi Ernst Haas, na década de 1980, que sugeriu uma reflexão sobre os processos de cooperação internacional baseados na aprendizagem, isto é, na introdução à política de conceitos científicos consensuais (HAAS, 1983). E. Adler e P. Haas ${ }^{5}$ desdobraram tal problematização em um programa de pesquisa alinhado geralmente à perspectiva construtivista e que ficou conhecido como "comunidades epistêmicas" (ADLER; HAAS, 1992).

Ao longo das duas últimas décadas ${ }^{6}$, os estudos internacionais revelaram a existência de inúmeras comunidades epistêmicas altamente influentes basicamente definidas como redes de especialistas - que estiveram envolvidas na trajetória da cooperação internacional em virtude de sua experiência profissional compartilhada (HAAS, 2015).

Apesar da concordância sobre a importância da ciência para a formulação de políticas públicas, ainda existem percepções divergentes de como, quando e em que condições a ciência influencia as políticas e, consequentemente, sobre a forma como a interação entre ciência e política deve ser melhor organizada (LIDSKOG; SUNDQVIST, 2015; KELLER, 2009). Estudos comparativos sobre regimes ambientais confirmaram que a experiência científica organizada tem uma influência notável sobre a eficácia dos regimes ambientais, principalmente quando os temas tratados nos acordos são altamente técnicos e envolvem grandes incertezas científicas (ANDRESEN, 2000; HAAS, 2006).

Haas (2004) argumenta que, através do desenvolvimento de uma comunidade epistêmica, a ciência pode desempenhar um papel importante na definição de decisões políticas. Ele enfatiza a importância da ciência e, em particular, do conhecimento consensual na formulação de políticas. Para o autor, a ciência baseada no consenso pode desempenhar um papel independente e importante influenciando e até mesmo reformulando os interesses dos Estados. Assim, da perspectiva da comunidade epistêmica, os regimes ambientais podem ser

5 Peter Haas iniciou seu trabalho com as comunidades epistêmicas quando ele estava fazendo sua dissertação sobre o Mar Mediterrâneo (1982-1983) (HAAS 1989; HAAS 1990). Através de entrevistas de elite com muitos membros envolvidos no debate de poluição do Mediterrâneo, ele notou que muitos deles apresentavam crenças compartilhadas e um consenso de conhecimento que influenciava suas decisões políticas.

6 O estudo das "comunidades epistêmicas" tornou-se um programa de pesquisa progressivo e, desde então, o conceito foi aplicado a numerosos exemplos (ver a International Organization Special Edition de 1992). Vinte anos depois, a abordagem foi revisada pela CROSS (2013), e ainda mais discutida por Peter Haas ao longo de sua carreira, tornando-se a referência principal para este programa de pesquisa específico (HAAS, 2014, 2015). 
impulsionados não só pelos poderes do Estado, mas também pelas redes epistêmicas sob certas condições, sendo que um fator não exclui o outro (HAAS, 2004).

O argumento central é que, normativamente, as comunidades epistêmicas fornecem melhores recomendações científicas do que outros modos de orientação política, uma vez que é provável que o parecer especializado tenha embasamento mais racional. Ao contrário de outros grupos de interesse organizados e ativos na formulação de políticas públicas, as comunidades epistêmicas têm crenças internas que as tornam mais propensas a fornecer informações que são politicamente inalteradas e, portanto, com maiores chances de que essa informação será enquadrada e seguida por autoridades políticas dada sua imparcialidade (HAAS, 2015).

Uma análise de comunidades epistêmicas assume que os tomadores de decisão, nesse caso, os Estados, constroem suas realidades políticas com base no conhecimento científico fornecido pelos especialistas. Efeitos esperados dessa relação levam os agentes a moldar ou modificar as ações de outros atores e, em consequência dessas ações, há benefícios sociais em virtude das ações coletivas.

Para Haas $(2015$, p. 157),

Comunidades epistêmicas é um conceito aplicado por estudiosos construtivistas da ciência política para analisar o processo pelo qual Estados e outros atores políticos formulam seus interesses e reconciliam diferenças de interesse. As comunidades epistêmicas são o principal canal pelo qual o conhecimento consensual sobre entendimentos causais é aplicado à coordenação de políticas internacionais e pelo qual os Estados podem aprender por meio de processos de cooperação internacional.

Esse entendimento fornece um excelente quadro de análise para discutir a eficácia dos acordos ambientais. A temática ambiental, em geral, opera sob condições de incerteza, onde os tomadores de decisão têm uma variedade de incentivos e razões para a consulta às comunidades epistêmicas, algumas mais politicamente motivadas que outras (HAAS, 1992). Esses novos atores atuando na arena internacional podem fazer com que os governos reconheçam e sigam novos interesses na proteção ambiental.

7 "epistemic communities is a concept applied by constructivist scholars of political science to focus analytic attention on the process by which states and other political actors formulate their interests and reconcile differences of interest. Epistemic communities are a principal channel by which consensual knowledge about causal understandings is applied to international policy coordination, and by which states may learn through processes of international cooperation”. 
A despeito de todos os esforços que diversos pesquisadores fizeram para abordar em suas pesquisas o conceito de comunidades epistêmicas, incluindo a segurança europeia (CROSS, 2015), a caça à baleia (PETERSON, 1992) e o Mediterrâneo (HAAS, 1990), a pesca internacional, objeto deste trabalho, ainda não foi explorada nesse quadro teórico-metodológico.

A pesca internacional é uma temática interessante para ser discutida no âmbito das comunidades epistêmicas, uma vez que lida com questões mergulhadas em incertezas científicas, de conteúdo altamente técnico e especializado, onde há muito interesse econômico e político por parte dos Estados envolvidos e atores não estatais desempenhando diversos papéis, e sem consenso na maioria das decisões.

Enquanto as comunidades epistêmicas podem estar entre os principais agentes responsáveis pela articulação de princípios, normas e regras, a extensão em que elas se difundem e são embutidas internacionalmente tem a ver com a influência política dos membros da comunidade epistêmica: sua capacidade de persuadir os outros, sua capacidade para consolidar a influência na burocracia institucional e ainda de manter a influência ao longo do tempo. Os interesses e decisões dos Estados para implantar o poder do Estado são, portanto, sujeitos a conhecimento consensual (HAAS, 2014).

É nesse contexto que se define a comunidade epistêmica como um grupo de profissionais, muitas vezes de disciplinas diferentes, que compartilham as seguintes características essenciais (HAAS, 2012, p. 351):

- crenças em princípios compartilhados (shared principled beliefs). Essas crenças são baseadas em valores para a ação social dos membros da comunidade;

- crenças causais compartilhadas ou julgamento profissional (shared causal beliefs or professional judgment). Tais crenças fornecem razões analíticas e explicações de comportamento, oferecendo explicações causais para as múltiplas ligações entre as possíveis ações políticas e os resultados desejados;

- noções comuns de validação (common notions of validity): critérios intersubjetivos, definidos internamente para validar o conhecimento. Isso permite que os membros da comunidade se diferenciem com confiança entre reivindicações garantidas e injustificadas sobre estados do mundo e políticas para mudar esses Estados;

- um grupo com política comum (a common policy enterprise): um conjunto de práticas associadas a problemas que devem ser abordados, presumivelmente, por convicção de que o bem-estar humano será melhorado como consequência. 
Não são numerosos os casos em que o poder tem ouvido a ciência para tomada de decisão no campo das negociações internacionais. Alguns exemplos positivos são os acordos que versam sobre o ozônio estratosférico e a chuva ácida europeia, amplamente saudados como entre os mais eficazes e maiores esforços de governança ambiental internacional da era contemporânea (HAAS 2001; MILES et al., 2002). Ou, ainda, o plano do Mediterrâneo para lidar com a poluição (HAAS, 1989). Por outro lado, há casos anômalos em que o argumento falhou, como desertificação e caça à baleia (HAAS, 2015).

De acordo com essa abordagem, a ciência influenciará os tomadores de decisão quando o desenho institucional do acordo permitir uma contribuição científica organizada e ainda incluir um grupo forte e isolado de especialistas que possuem um “conhecimento útil”. Embora o conhecimento utilizável tenha sido usado em diferentes contextos e situações, o termo engloba um núcleo substantivo que o torna útil para os formuladores de políticas e uma dimensão processual que fornece um mecanismo para a sua transmissão da comunidade científica para o mundo das políticas (HAAS; STEVENS, 2011).

Em um contexto de governança ambiental global, a contribuição científica é comumente disponibilizada através de painéis científicos, que fazem parte dos acordos internacionais. Esses painéis são estabelecidos pelos Estados para fornecer conhecimento e informação sobre riscos globais e transfronteiriços. Embora as tarefas específicas variem de acordo para acordo, em geral, os painéis cumprem tarefas como compilar conhecimento sobre o estado da arte de um tópico específico e identificar opções de políticas para solucionar problemas compartilhados. Funcionalmente, eles têm como objetivo contribuir com o conhecimento e promover a aprendizagem através do fornecimento de informação (HAAS, 2017).

Os painéis científicos são formados por especialistas que se reúnem periodicamente e produzem conhecimento através de relatórios para instituições internacionais ou para os comitês organizadores de regimes internacionais. Estudos descritivos sobre painéis científicos descobriram que eles são caracterizados por um pequeno número de características arquitetônicas regulares (HAAS; STEVENS, 2011), porém, as formas e estratégias utilizadas para que esses objetivos sejam alcançados variam. Alguns se reúnem regularmente, outros com pouca frequência. Alguns são comitês permanentes, enquanto outros são ad hoc. Os especialistas podem ser nomeados e selecionados por secretarias, pelos Estados membros ou pelas Conferências das Partes (COPs) (Quadro 1). 
O trabalho normativo mais recente ajudou a projetar uma taxonomia de diferentes tipos de painéis científicos e suas contribuições potenciais para a governança global (HAAS; STEVENS, 2011). Já o trabalho comparativo sobre avaliações ambientais globais descobriu que os painéis científicos organizados nos níveis nacional e internacional influenciaram significativamente as negociações ambientais e contribuíram para a sua eficácia através da provisão de "conhecimento utilizável" (usable knowledge). O conhecimento utilizável é a forma substantiva de conhecimento que os cientistas e as comunidades epistêmicas oferecem e que ajuda a ressaltar a natureza do conhecimento. Essa forma de conhecimento envolve a informação fornecida pela comunidade científica através de instituições: deve ser crível, legítimo e saliente.

CLARK e MAJONE (1985) fizeram uma reflexão sobre a relação entre cientistas e tomadores de decisão e por que, às vezes, ambos se mostram insatisfeitos uns com os outros. Para produzir conhecimentos científicos utilizáveis, eles definiram alguns critérios importantes, tais como adequação, valor, legitimidade e eficácia: a adequação se relaciona com a inclusão de todos os conhecimentos ou fatos relevantes para o assunto em questão; o valor tem a ver com a contribuição para uma maior compreensão e para políticas significativas; a legitimidade relaciona-se com a aceitação do conhecimento por parte de outros que não a comunidade que o desenvolveu; a eficácia relaciona-se com sua capacidade de moldar a agenda ou avançar o estado do debate e, finalmente, melhorar a qualidade do meio ambiente. $\mathrm{O}$ fato é que um cientista não tem tudo isso em mãos o tempo todo, e acaba por não satisfazer, sempre, o tomador de decisão que busca respostas práticas, racionais e com o maior nível de exatidão possível.

Além disso, os critérios de legitimidade científica para a tomada de decisões institucionais são: precisão (amplamente percebida como verdadeira); legitimidade (obtida através de processos imparciais isolados da influência política direta); e saliência (politicamente relevante e atraente). A ciência não é bem-sucedida em influenciar a política quando há: suspeita pública sobre seu desenvolvimento ou metodologia; problemas de desenho institucional; falta de clareza nas prioridades de avaliação científica; e a não cessão de "autoridade” por parte do governo à ciência, principalmente caso as decisões políticas resultantes tenham consequências desfavoráveis a algum projeto político (HAAS; STEVENS, 2011). 


\section{A eficácia da cooperação internacional}

A discussão sobre a eficácia da cooperação internacional é complexa por vários motivos. Uma das razões é a dificuldade em definir o que se entende por eficácia e quais são os fatores mais qualificados para se medir e/ou analisar seu efeito. Para Young (1999), os regimes podem variar ao longo de um contínuo de arranjos ineficazes, que são apenas "acordos de papel”, para arranjos altamente efetivos, que produzem soluções rápidas e decisivas para os problemas em questão.

Em alguns casos, os acordos foram efetivos na mudança de comportamento e, então, acabaram por impactar o meio ambiente de forma positiva (HAAS, 1992).

Em outros casos, o sucesso - seja na criação de mecanismos internacionais, seja em sua influência no comportamento do Estado ou dos indivíduos, ou em sua capacidade de melhorar as condições do meio ambiente - é menos certo. No entanto, é através dessas experiências que podemos encontrar lições para aprender a tornar a cooperação internacional mais efetiva e, ainda mais importante, para entender como são os acordos mais eficazes.

Há muitas abordagens que se poderiam utilizar para estudar a eficácia de um acordo internacional. Para Young (1999), pode ser uma abordagem política, normativa, econômica, legal e de resolução de problemas. Cada uma delas apresenta prós e contras, e podem ser usadas analiticamente, separadas ou em grupos, dependendo do objetivo da pesquisa.

Para os fins desta pesquisa, arranjos eficazes serão aqueles que implicam mudanças por parte dos Estados e que levam ou podem conduzir a melhorias na qualidade ambiental. Em outras palavras, a eficácia aqui significa que o conhecimento das comunidades epistêmicas induziu Estados a mudarem seu comportamento de forma a promover a conquista de aspirações negociadas, especialmente para a recuperação dos estoques pesqueiros.

Em geral, as OROPs têm como objetivo estabelecer medidas de conservação e manejo da pesca, portanto, a abundância atual dos estoques de peixe (biomassa) parece ser um bom critério para medir a eficácia de um acordo em termos de solução de problemas. Existem 18 OROPs com mandatos para estabelecer medidas de conservação e manejo da pesca, o que significa que quase todo o alto-mar está coberto por pelo menos uma OROP. No entanto, a eficácia das OROPs raramente foi avaliada de forma abrangente, apesar das indicações do declínio de muitas unidades populacionais de peixes do alto-mar (FAO, 2016). Reconhecendo os 
desafios em medir quantitativamente a eficácia dos acordos, essa pesquisa optou por basear-se em Cullis-Suzuki e Pauly (2010), que avaliaram a eficácia das 18 OROPs do mundo8, e então produziram um ranking de eficácia, utilizando principalmente como critério a recuperação dos estoques pesqueiros. Assim, selecionando 3 casos, com posições variadas no ranking, será possível discorrer sobre os elementos que conferem maior ou menor eficácia.

O ranking com pontuações produzido por Cullis-Suzuki e Pauly (2010) pontuou: CCAMLR (100\%), ICCAT $(37,5 \%)$ e CCSBT $(0,0 \%)^{9}$, e para se ter uma base comparativa, esses foram os acordos analisados na presente pesquisa. Cullis-Suzuki e Pauly (2010) reconhecem e concluem que as OROPS, de uma forma geral, não têm sido bem sucedida em impedir que muitas populações de peixe entrem em declínio, embora seja também parte de suas atribuições a conservação dessas populações. E deixam em aberto um questionamento sobre o porquê desse fracasso no manejo das espécies.

Existem muitas abordagens na área das relações internacionais que permitem uma resposta coerente à questão deixada pelos autores; esta pesquisa buscará respostas particularmente na abordagem da comunidade epistêmica e na discussão sobre a interação entre a ciência e a política.

Haas (1992) argumenta que a difusão de novas ideias e informações é um dos fatores que pode levar a novos padrões de comportamento, sendo um determinante importante de eficácia da cooperação política internacional. Além disso, para Haas, Keohane e Levy (1995, p. 19), três principais condições podem contribuir para o sucesso do manejo de problemas ambientais através de instituições. O primeiro é que a preocupação governamental deve ser suficientemente alta para mobilizar os Estados a ponto de disponibilizarem recursos, já escassos, para resolver o problema ambiental. Segundo, os problemas ambientais comuns e transfronteiriços não podem ser efetivamente resolvidos sem um ambiente contratual em que os Estados façam compromissos que sejam capazes de cumprir e honrar. E, por

8 Existem 18 OROPs e potenciais com mandatos para estabelecer medidas de conservação e manejo da pesca, o que significa que quase todo o alto-mar global está agora coberto por pelo menos uma OROP. No entanto, a eficácia das OROPs raramente foi avaliada de forma abrangente, apesar das indicações do declínio de muitas unidades populacionais de peixes do alto-mar (FAO, 2014).

9 Os acordos serão referidos nesse trabalho através de sua sigla em inglês, tais como: Convenção sobre a Conservação dos Recursos Vivos Marinhos Antárticos (CCAMLR), Convenção Internacional para a Conservação dos Atuns e Afins (ICCAT) e a Convenção para a Conservação do Atum do Sul (CCSBT), uma vez que não há siglas pré-estabelecidas em português. 
último, mas não menos importante, que os Estados possuam capacidades política e administrativa para fazer os ajustes domésticos necessários para a implementação do acordo.

Ainda para Haas e Stevens (2011), o conhecimento opera e muda o comportamento quando organizado e transmitido de forma que os formuladores de políticas possam entender e confiar na informação. Nesse contexto, regimes que são desenvolvidos por um processo de aprendizagem social e cujas regras refletem o consenso científico sobre sustentabilidade ambiental tendem a ser mais eficazes.

\section{As OROPs e a interface entre ciência e política}

A interface entre o conhecimento científico e a política para o manejo pesqueiro tem uma longa história, pelo menos em teoria. O artigo 61 da Convenção das Nações Unidas sobre o Direito do Mar (UNCLOS, 1982) demanda que as decisões levem em consideração “o melhor conhecimento científico disponível”, além disso, a necessidade de aconselhamento científico para a tomada das decisões e para o estabelecimento de uma nova OROP foi também confirmada pelo Acordo de Estoque Pesqueiros das Nações Unidas (UNFSA, 1995).

A ciência é, portanto, evocada para transmitir mensagens de que as decisões tomadas levaram em conta todas as informações relevantes, que a informação foi utilizada de maneira sistemática e sólida, de maneira objetiva, verificável e equilibrada, levando, consequentemente, a decisões racionais, dada a informação disponível (PIELKE, 2004). No entanto, "baseado na ciência” não significa que todas as fontes e tipos de informação tenham tido igual peso nas decisões, nem que a "informação científica" constituiu o único fator considerado na tomada de decisões (MITCHELL et al., 2006).

Mora et al (2009) estudou a eficácia dos regimes de manejo da pesca em todo o mundo e também calculou a provável sustentabilidade das capturas relatadas para determinar como o manejo afeta a sustentabilidade da pesca quando o conhecimento científico é aplicado. Seus resultados alegaram que a conversão do conselho científico em políticas, através de um processo participativo e transparente, é o cerne da obtenção da sustentabilidade da pesca. No entanto, apenas alguns países têm uma base científica sólida para recomendações de manejo; 
processos transparentes e participativos para converter essas recomendações em políticas; e, que ao mesmo tempo, capacidade de assegurar o cumprimento dos regulamentos. Assim, a incerteza também desempenha um papel importante nesse cenário de pesca.

O desenho dos processos de criação de conhecimento e o acesso aos seus resultados são importantes para a utilidade e a credibilidade da governança (RIDGEWAY, 2014). Com o envolvimento de contribuições científicas organizadas para o processo político, os resultados negociados são muito mais propensos a produzir esforços eficazes do que os meros compromissos políticos (HAAS, 2006). A natureza e o valor do aconselhamento científico fornecido às OROPs são moldados por variáveis independentes que incluem os arranjos institucionais e operacionais estabelecidos pela própria OROP, além disso, conta com a qualidade e a temporalidade dos dados subjacentes à informação fornecida (WILLOCK; LACK, 2006).

A necessidade da contribuição científica na governança ambiental global tem sido amplamente reconhecida desde a Conferência das Nações Unidas para o Meio Ambiente Humano de 1972 (CONTINI; SAND, 1972). Os painéis científicos internacionais foram, então, sistematicamente anexados aos regimes ambientais internacionais, embora com resultados mistos (HAAS, 2017).

Para HAAS (2017) 10, "Painéis científicos são organizações que unem política e ciência, bem como relacionam a ciência à governança política de problemas compartilhados". Através dos painéis científicos, o conhecimento consensual persuade os governos a mudarem suas práticas em nome dos objetivos coletivamente negociados. O mecanismo social pelo qual os painéis científicos influenciam a eficácia do regime é a aprendizagem. Uma vez que os Estados mudam voluntariamente seu comportamento com base no conhecimento dos painéis científicos, os painéis científicos operam através do exercício da influência social.

Os três acordos selecionados para essa pesquisa têm seu desenho institucional construído conforme descrito na Quadro 1 (adaptado e atualizado a partir de Haas e Stevens, 2011).

10 "Science panels are boundary organizations which bring together politics and science, as well as relate science to the political governance of shared problems" 


\section{Quadro 1. Informações sobre o desenho institucional para a ciência} para os acordos selecionados

\begin{tabular}{|c|c|c|c|}
\hline & CCAMLR & ICCAT & CCSBT \\
\hline Objetivo da Convenção & $\begin{array}{c}\text { A conservação } \\
\text { dos recursos vivos } \\
\text { marinhos da } \\
\text { Antártida }^{11}\end{array}$ & $\begin{array}{c}\text { Manter as } \\
\text { populações de } \\
\text { peixes em níveis que } \\
\text { permitam a captura } \\
\text { máxima sustentável }\end{array}$ & $\begin{array}{l}\text { Garantir, através } \\
\text { de uma gestão } \\
\text { adequada, a } \\
\text { conservação e } \\
\text { otimização da } \\
\text { utilização do } \\
\text { atum-azul do sul }\end{array}$ \\
\hline Organização do comitê & $\begin{array}{c}\text { Permanente; } \\
\text { estabelece a } \\
\text { agenda }\end{array}$ & Ad hoc & Permanente \\
\hline Seleção de Especialistas & Estados & Estados & Estados \\
\hline Tipos de comitê científico & Aberto & Aberto & Aberto \\
\hline Decisões tomadas por & Consenso & Maioria & Consenso \\
\hline $\begin{array}{c}\text { Decisões (vinculantes/ } \\
\text { aconselhamento) }\end{array}$ & Obrigatório & Obrigatório & Obrigatório \\
\hline Objeção às decisões & Não & Sim & Sim \\
\hline Grau de aprendizagem social & $\begin{array}{c}\text { Sim, há } \\
\text { aprendizagem } \\
\text { social - acontece } \\
\text { através de seu } \\
\text { próprio desenho } \\
\text { institucional }\end{array}$ & $\begin{array}{c}\text { Intermediário - } \\
\text { pode acontecer, } \\
\text { mas depende muito } \\
\text { do envolvimento } \\
\text { de outras partes } \\
\text { interessadas para } \\
\text { criar um ambiente } \\
\text { onde o conhecimento } \\
\text { seja justificado }\end{array}$ & $\begin{array}{c}\text { Pouco/nenhum } \\
\text { - melhorou com } \\
\text { o tempo e com } \\
\text { a aprovação do } \\
\text { Procedimento de } \\
\text { Manejo }\end{array}$ \\
\hline Dados fornecidos por & Governo & Governo & Governo \\
\hline
\end{tabular}

Fonte: adaptado e atualizado de Haas e Stevens, 2011.

Nota-se que o desenho institucional para esses acordos é muito semelhante e, em geral, não é voltado para conseguir isolar os órgãos científicos da influência política direta ou indireta. O desenho dos comitês científicos para políticas de pesca reflete uma patologia institucional que limita a capacidade da ciência em influenciar o poder, no entanto, em diferentes níveis e em diferentes situações.

11 Para o propósito dessa convenção, o termo "conservação" inclui uso racional. 
O site da CCAMLR mostra que esse acordo busca dar prioridade à ciência na tomada de decisão e afirma que "a ciência é fundamental para a CCAMLR". O próprio texto da convenção exige que a comissão tome as decisões levando em conta totalmente as recomendações e conselhos do comitê científico. Essa ênfase na ciência foi reiterada em 2009 na Resolução 31/XXVIII (CCAMLR, 2009) sobre o uso da "melhor ciência disponível".

Não por acaso a CCAMLR vem sendo considerada como uma das OROPs mais eficazes e capazes de demonstrar bons resultados, de acordo com o ranking produzido por Cullis-Suzuki e Pauly (2010) em termos de recuperação de biomassa (variável dependente). Haas (2006) e outros pesquisadores (CONSTABLE, 2011; BROOKS, 2013; BROOKS et al., 2014) também corroboram que a CCAMLR é um bom exemplo de um acordo; os estoques de peixes estão se recuperando e os padrões focados em questões ambientais foram aprimorados, adotados e implementados. Conforme apontado por BROOKS et al., (2014), uma das razões do sucesso da CCAMLR ao passar por mais medidas de conservação e baseadas em ecossistemas é que ela é composta por membros com foco na ciência.

No entanto, Constable (2011) observou que, embora a CCAMLR tenha contribuído com a recuperação da população de peixes e seja considerada um acordo eficaz, ainda é necessário maior consenso nas recomendações científicas, dentro do seu próprio comitê científico (CONSTABLE, 2011). Isso significa que existe um desenho institucional que promove o fluxo de ciência para os decisores em seu organograma, onde o comitê científico está diretamente abaixo da própria comissão, mas, devido à falta de acordo entre os cientistas, não há consenso sobre a informação. Existe uma falta de conhecimento utilizável (usable knowledge) para proporcionar uma experiência de aprendizagem social ainda maior do que a que já ocorre.

Quando a CCAMLR é comparada à ICCAT e à CCSBT, mesmo com essas críticas e a necessidade de melhorias, a CCAMLR é o acordo que apresenta mais políticas de aprendizagem social e, consequentemente, a mais eficaz em termos de resolução de problemas. Isso corrobora Cullis-Suzuki e Pauly (2010), onde CCAMLR marcou (Q score) 100\% em seu desempenho.

Já a ICCAT é conhecida historicamente por não ouvir a ciência (KVIST, s/d). O atum-azul do Atlântico ajuda a ilustrar o baixo nível de aprendizagem social da ICCAT, já que no passado eles ignoraram constantemente o conselho de seus cientistas sobre essa espécie. A comissão optou por adotar regulamentos sem impô-los, então, em 1974, a ICCAT aprovou uma recomendação vinculativa 
para limitar as capturas nos “níveis atuais” de acordo com o parecer científico. A comissão estabeleceu por muitos anos níveis de captura superiores aos recomendados pelo seu próprio comitê científico (GONÇALVES, 2016).

Embora o conselho do comitê científico da ICCAT tenha se tornado mais otimista, a diferença entre capturas e aconselhamentos científicos foi notável, como ilustrado por Webster (2011). Em 2011/2013, houve uma mudança, quando a comissão, pela primeira vez, aceitou a quota de sugestão do comitê científico para o atum-azul do Atlântico (GONÇALVES, 2016). A decisão foi bem vista, mesmo por ONGs internacionais que muitas vezes se queixaram da falta de ciência na ICCAT (CRESSEY, 2012; WWF, 2012).

Como destacado por Aranda, De Bruyn e Murua (2010) na sua revisão sobre a OROP, a pesquisa e avaliação de cada OROP depende da sua estrutura científica. No caso da ICCAT, a contribuição científica vem dos seus grupos de trabalho, compostos por cientistas que representam os Estados membros. Os dados para o processo científico são geralmente fornecidos pelos Estados membros, incluindo dados totais de captura, captura e esforço, e dados de captura e tamanho. No entanto, a apresentação de dados é muitas vezes incompleta ou tardia e pode até ser subestimada, o que compromete os dados utilizados para estimativas científicas. Além disso, eles acrescentaram que os dados operacionais detalhados raramente são fornecidos por membros e são considerados altamente confidenciais.

Para a CCSBT, o comitê científico considera análises de avaliação de estoque realizadas por cientistas e consultores nacionais e é auxiliada por um presidente independente do comitê científico e por um painel de especialistas independentes. O papel desse painel é facilitar o consenso, e se isso revelar-se impossível, fornecer sua própria visão independente à comissão (ARANDA, DE BRUYN E MURUA, 2010). A prestação de pareceres científicos independentes é fundamental para o funcionamento das OROPs, mesmo que as mesmas sejam, em grande parte, instrumentos políticos. As pressões políticas e a intervenção na ciência, bem como o cruzamento das fronteiras entre os processos científicos e políticos, no entanto, parecem ser relativamente comuns nessa arena (POLACHECK, 2012).

A maioria dos acordos que possuem algum nível de aprendizagem social tem uma comissão permanente e, junto a isso, comitês de pesquisa para fornecer alertas oportunos sobre novos problemas, monitorar realizações de objetivos para o regime e educar políticos e formuladores de políticas sobre questões ambientais (HAAS; STEVENS, 2011). Isso traria uma característica adaptativa ao acordo. Mas este não é o caso dos acordos de pesca estudados aqui, pois todas as informações 
científicas são fornecidas pelos órgãos governamentais que nem sempre são capazes de fornecer informações precisas, e nem são capazes de fornecer informações ao longo de um tempo suficiente para a elaboração de tendências. Ao analisar as respectivas avaliações de desempenho (CCAMLR, 2008; ICCAT, 2009 e CCSBT, 2008), é possível ver que a CCAMLR tem menos dependência de informações do governo do que as demais, o que contribui para seu melhor desempenho.

A manutenção e o apoio a organismos científicos dentro de acordos multilaterais de governança ambiental são absolutamente vitais para a construção de conhecimentos utilizáveis dentro do regime. Painéis científicos permanentes permitem a constante construção e transmissão de informações precisas e oportunas. Três tipos de estruturas de comissão permanente são típicos dos regimes ambientais multilaterais (HAAS; STEVENS, 2011).

O primeiro tipo inclui grupos científicos que estabelecem seus próprios horários e agendas de pesquisa. Esses grupos se encontram como acham conveniente durante a plenária política, de acordo com suas próprias necessidades. Esse é o caso do comitê científico da CCAMLR. O comitê científico se reúne anualmente e imediatamente antes da reunião da comissão. A fim de abordar a vasta gama de áreas científicas que podem afetar as decisões da comissão, o comitê científico estabeleceu uma série de grupos de trabalho que se reúnem durante o ano; eles estabelecem sua própria agenda e ajudam na formulação de pareceres científicos sobre áreas chave.

O segundo tipo é composto por aqueles grupos cujas reuniões são definidas pela comissão. Por exemplo, a maioria das comissões exige que seus órgãos científicos se reúnam um mês ou dois meses antes da plenária, como é o caso da ICCAT e CCSBT, por exemplo.

Finalmente, o terceiro tipo inclui painéis de ciência ad hoc, que, especialmente, se solicitado pelos Estados membros, introduzem um alto envolvimento político na divulgação de ciência, que também coincide com o desenho da ICCAT. A CCSBT fez progressos na tentativa de proteger a ciência da política, estabelecendo um painel consultivo para fornecer contribuições externas para a avaliação de estoque e os processos científicos. Também nomeou um presidente independente para o comitê científico, neutro, que não representa nenhuma visão governamental, e nem representa nenhum governo.

Além disso, outro fator que pode ser avaliado é a escolha dos cientistas. De acordo com Haas e Stevens (2011), os acordos mais bem-sucedidos possuem uma seleção de cientistas de outras secretarias ou mesmo de órgãos científicos 
de outras organizações intergovernamentais. Esse não é o caso de nenhum dos acordos discutidos aqui. Todos os cientistas que são parte do comitê científico são designados pelos próprios países, o que não garante a salvaguarda de informações científicas.

Nos acordos de pesca aqui selecionados, o desenho de um organismo científico está aberto, o que permite que cada país membro nomeie um representante para um órgão científico, sem que necessariamente ele possua um background científico. Isso geralmente não cria salvaguardas adequadas entre os órgãos científicos e os tomadores de decisão.

A partir dessa pesquisa, é possível aprender que a CCAMLR tem o melhor desenho institucional para a ciência e que permite que a ciência e a comunidade epistêmica produzam e influenciem as decisões, suas ideias e conhecimentos. Algumas dessas características podem ser compartilhadas com outros acordos, a fim de melhorar as boas práticas para a influência da ciência sobre decisões políticas. No entanto, parece que a comunidade científica ainda não possui uma voz única, e essa dissonância e falta de consenso evitam um nível ainda maior de eficácia. A falta de consenso promove a incerteza, que é uma das principais razões pelas quais as ações de longo prazo, como a proteção da biodiversidade ou a reconstrução da população de peixes, enfrentam dificuldades de implementação.

De acordo com a revisão do desempenho da ICCAT (ICCAT, 2009), enquanto a modelagem e a avaliação de estoque não são uma ciência perfeita, as pescarias manejadas pela ICCAT são razoavelmente bem compreendidas e o comitê científico é considerado bem profissional. No entanto, a falta de participação e a falta de dados fornecidos pelos países podem provocar sérios danos à qualidade científica.

Já quanto à revisão do desempenho do CCBST (2008), o processo atual para desenvolver e fornecer pareceres científicos sobre o atum-azul do comitê científico estendido é um excelente modelo que ajudou a melhorar a integridade do processo científico da CCSBT. O acesso a cientistas nacionais altamente competentes está disponível e se reflete na abundância e qualidade dos trabalhos científicos apresentados aos vários fóruns científicos da CCSBT. Os arranjos de painel e presidente independente acrescentaram, em 1999, uma maior confiabilidade nos dados, e ainda uma dificuldade maior para os governo imporem suas agendas políticas.

No entanto, a CCSBT possui disposições específicas dentro das suas regras de procedimento para a confidencialidade dos documentos apresentados às reuniões. 
O acesso público aos documentos científicos utilizados na tomada de decisão pública é considerado um princípio fundamental, segundo o qual a ciência deve e é conduzida dentro da OROP (por exemplo, UNFSA). O mesmo acontece, em geral, em muitas sociedades (por exemplo, com as leis de liberdade de informação) (POLACHECK, 2012).

Não há dúvida de que a falta de transparência, nesse caso, diminui a representatividade da informação e destrói uma chance única de criar uma política baseada em ciência com conhecimento e confiança.

O que é notável, ao olhar para os órgãos científicos da CCAMLR, ICCAT e CCSBT e para as instituições de pesquisa que os mesmos representam, é a presença de cientistas de muito alto calibre e competência. A diferença entre eles é o nível de dependência da informação dos países membros. A robustez do sistema pode ser aumentada ao reduzir essa dependência através de um maior investimento coletivo em programas científicos para coletar e compilar informações independentes.

\section{Conclusões}

Esta pesquisa conclui que, principalmente quando se trata de temas altamente técnicos, mergulhados em incertezas científicas, e com o envolvimento de diversos interesses econômicos e políticos, é importante que o desenho do acordo favoreça a formação de conhecimento científico legítimo, útil e isolado da política. No entanto, em grande medida, a escolha, a qualidade e a diversidade dos conhecimentos envolvidos nos grupos de trabalho e na comunidade científica dependem das contribuições, do envolvimento e da vontade dos países.

Nos últimos anos, houve um reconhecimento crescente da necessidade das OROPs melhorarem o seu desempenho devido às demandas contidas nos recentes acordos internacionais destinados a uma melhor conservação e gestão dos recursos pesqueiros. O desenho institucional formal das OROPs analisadas neste artigo não permite aos cientistas produzir conhecimento utilizável (usable knowledge), conforme definido por Clark e Majone (1985) e demonstrado empiricamente, para alguns acordos, no capítulo Haas e Stevens (2011) e ainda em Haas (2017). Nos acordos aqui apresentados, a ciência não é totalmente isolada da influência política, o que pode gerar uma falta de confiança nos tomadores de decisão.

Como pôde ser verificado, até o momento, a CCAMLR apresenta um grupo de fatores mais consolidados que podem garantir que o conhecimento contribua 
para influenciar a política e produzir para um maior nível de aprendizagem social. Potencialmente, esses fatores podem garantir um maior desempenho na recuperação de biomassa, como mostram Cullis-Suzuky e Pauly (2010). Por outro lado, a ICCAT e a CCSBT precisam ainda encontrar um caminho para isolar e fortalecer seus painéis científicos, a fim de capacitá-los a produzir informações mais independentes, precisas e legítimas para melhorar sua aprendizagem social e, em consequência, a eficácia na recuperação de populações de peixes.

Essa pesquisa corrobora Haas (2004), ao afirmar que o conhecimento pode influenciar a tomada de decisão e, com isso, viria mais eficácia em termos de resolução de problemas. No entanto, para que funcione para os acordos de pesca, é ainda necessário que os conhecimentos e as reivindicações sejam desenvolvidos isoladamente da política; algo que ainda não está previsto nas regras formais ou nem sempre é exigido nos acordos aqui estudados.

É importante considerar que, para além da necessidade de serem produzidos acordos e regimes que tratem de questões complexas e transnacionais de forma a propiciar o fluxo de conhecimento, e ainda para além da importância de ser produzido conhecimento considerado legítimo pelos tomadores de decisão, existem outras variáveis que também influenciam no processo de conferir maior eficácia à governança global, e que passam por escolhas racionais em suas vertentes econômicas e políticas.

\section{Referências}

ADLER, E., \& HAAS, P. M. 1992. Conclusion: epistemic communities, world order, and the creation of a reflective research program. International Organization, 46 (1), 367-390.

ANDRESEN, S. (2000). Science and Politics in International Environmental Regimes: Between Integrity and Involvement. Issues in Environmental Politics. New York: Manchester University Press.

ANDRESEN, S. (2016). International regime effectiveness. IN: Falkner, R. (2016). The handbook of global climate and environment policy. John Wiley \& Sons. 596pp. ARANDA, M., DE BRUYN, P. , \& MURUA, H. (2010). A report review of the tuna RFMOs: CCSBT, IATTC, IOTC, ICCAT and WCPFC. EU FP7 Project, (212188), 171.

BIERMAN, F. (2008). Earth System Governance: A Research Agenda. In: Institutions and Environmental Change: Principal Findings, Applications, and Research Frontiers (Eds: Young, O.R; King, L.A.; Schroeder, H). Cambridge, MA: MIT Press. 
BROOKS, C. M. (2013). Competing values on the Antarctic high seas: CCAMLR and the challenge of marine-protected areas. The Polar Journal, 3(2), 277-300.

BROOKS, C. M., WELlER, A. J. B., GJERDE, B. K., SUMAILA, C. U. R., ARDRON, D. J., BAN, E. N. C.,.. \& HALPIN, J. P. (2014). Challenging the” Right to Fish'in a Fast-Changing Ocean. Stan. Envtl. LJ, 33, 289-457.

CCAMLR (2008). CCAMLR performance review panel. Disponível em: < https://www. ccamlr.org/en/system/files/e-Prfrm \%20Review\%20Report \%20Jun09_0.pdf > . Acesso em: Abril, 2018.

CCAMLR (2009). Resolution on Best Available Science. Disponível em: < https://www. ccamlr.org/en/resolution-31/xxviii-2009 > . Acesso em: Abril, 2018.

CCSBT (2008). CCSBT performance review panel. Disponível em: (2008). < https://www. ccsbt.org/sites/default/files/userfiles/file/docs_english/meetings/meeting_reports/ ccsbt_15/PerformanceReview_IndependentExpertsReport.pdf > . Acesso em: Abril, 2018.

CLARK, W. C., AND MAJONE, G. (1985). The Critical Appraisal of Scientific Inquiries with Policy Implications. Science, Technology, \& Human Values 10 (3): 6-19.

CONSTABLE, A. J. (2011). Lessons from CCAMLR on the implementation of the ecosystem approach to managing fisheries. Fish and Fisheries, 12(2), 138-151.

CONTINI, P. \& P. H. SAND (1972). "Methods to Expedite Environmental Protection: International Econstandards.” American Journal of International Law 66(1): 37-59.

CRESSEY, D. (2012). Conservationists claim victory for science over tuna quotas. Disponível em: < http://blogs.nature.com/news/2012/11/conservationists-claimvictory-for-science-over-tuna-quotas.html > . Acesso em: Abril, 2018.

CROSS, MAI'A K. DAVIS (2013). Rethinking epistemic communities twenty years later. Review of International Studies, 39, pp 137-160.

CROSS, MAI’A, K. (2015). The Limits of Epistemic Communities: EU Security Agencies. Politics and Governance, 3(1), 90-100.

CULLIS-SUZUKI, S., \& PAULY, D. (2010). Failing the high seas: A global evaluation of regional fisheries management organizations. Marine Policy, 34:5, 1036-1042.

DESOMBRE, E. R. (2007). The global environment and world politics. Bloomsbury Publishing USA.

DINGWERTH, Klaus; PATTBERG, Philipp. Global governance as a perspective on world politics. Global governance: a review of multilateralism and international organizations, v. 12, n. 2, p. 185-203, 2006.

DUFFIELD, Mark. Global governance and the new wars: The merging of development and security. Zed Books Ltd., 2014.

FAO (Food and Agriculture Organisation). (2016). "The State of World Fisheries and Aquaculture” (2016), Disponível em: < http://www.fao.org/fishery/sofia/en > . Acesso em: Abril 2018. 
FORMAN, S., \& SEGAAR, D. (2006). New coalitions for global governance: the changing dynamics of multilateralism. Global Governance: A Review of Multilateralism and International Organizations, 12(2), 205-225.

GONÇALVES, L. R. (2016) Regional Fisheries Management Organizations: is power listening to Science? Tese (Doutorado em Relações Internacionais) - Instituto de Relações Internacionais, University of São Paulo, São Paulo, 2016. doi:10.11606/T.101.2016. tde-21112016-164951. Acesso em: 2017-12-21.

HAAS, E. B. (1983). Regime decay: conflict management and international organizations, 1945-1981. International Organization, v. 37, n. 02, p. 189- 256, 1983.

HAAS, P. M. (1989). Do Regimes Matter? Epistemic Communities and Mediterranean Pollution. International Organization 43. No. 3.

HAAS, P. M. (1990). Saving the Mediterranean: The Politics of International Environmental Cooperation, the Political Economy of International Change. New York: Columbia University Press.

HAAS, P. M. (1992). Introduction: Epistemic communities and international-policy coordination-introduction. International Organization 46(1). Special Edition. 1-35pp.

HAAS, P. M. (2001). Epistemic Communities and Policy Knowledge. In International Encyclopedia of Social and Behavioral Sciences, ed. N. J. Smelser, James Wright, and P. B. Baltes, 11578-11586. New York: Elsevier. 158

HAAS, P. M. (2004). When Does Power Listen to Truth? A Constructivist Approach to the Policy Process. Journal of European Public Policy 11 (4): 569-592.

HAAS, P. M. (2006). Evaluating the Effectiveness of Marine Governance. Prepared for the Nippon Foundation Task Force on the Dynamics of Regional Cooperation on Oceans and Coasts. 34pp.

HAAS, P. M. (2012). Epistemic Communities. IN: The Oxford Companion of Comparative Politics. Vol. 1 Oxford: Oxford University Press pp 351-359. Edited by Joel Krieger. DOI: $10.1093 /$ acref/9780199738595.001.0001.

HAAS, P. M. (2014). Reconstructing Epistemic Communities. Prepared for delivery at the 2014 Annual Meeting of the American Political Science Association, August 28-31, 2014.

HAAS, P. M. (2015). Epistemic communities, constructivism, and international environmental politics. Routledge.

HAAS, P. M. (2017). Coupling science to governance: straddling the science-policy interface. In: LITTOZ-MONNET, Annabelle (Ed.). The Politics of Expertise in International Organizations: How International Bureaucracies Produce and Mobilize Knowledge. Taylor \& Francis, 2017.

HAAS, P. , \& STEVENS, C. (2011). Organized science, usable knowledge and multilateral environmental governance. IN: Governing the Air: The Dynamics of Science, Policy, and Citizen Interaction, 125. 
HAAS, P. M.; KEOHANE, R.O. AND LEVY, M.A. (1995). The effectiveness of International Environmental Institutions". IN: Institutions for the Earth: sources of effective international environmental protection, edited by Haas, Keohane and Levy. 3-24. Cambridge, MIT Press.

ICCAT (2009). Report of the Independent Performance review of ICCAT. Disponível em: www.iccat.int/Documents/Other/PERFORM_\%20REV_TRI_LINGUAL.pdf > . Acesso em: Abril, 2018.

INOUE, Cristina Yumie Aoki. (2003). Regime global de biodiversidade. Comunidades epistêmicas e experiências locais de conservação e desenvolvimento sustentável: o caso Mamirauá. Brasília. Tese (Doutorado em Desenvolvimento Sustentável) - Centro de Desenvolvimento Sustentável, Universidade de Brasília, v. 348, p. 30-49, 2003.

KARNS, M.; KAREN, M. (2004). The politics and processes of global governance. Colorado: Lynne Rienner Publishers, 2004.

KELLER, A. C. (2009). Science in environmental policy: The politics of objective advice. MIT Press.

KVIST, S. Institutional fragmentation in fisheries management. (no date). Disponível em: < https://goo.gl/YyfTvw > .

LIDSKOG, Rolf; SUNDQVIST, Göran. When does science matter? International relations meets science and technology studies. Global Environmental Politics, v. 15, n. 1, p. 1-20, 2015.

MILES, EDWARD L, ARILD UNDERDAL, STEINAR ANDRESEN, JØRGEN WETTESTAD, JON BIRGER SKJAERSETH, AND ELAINE M. CARLIN. (2002). Environmental Regime Effectiveness: Confronting Theory with Evidence. Cambridge, MA: MIT Press.

MITCHELL, RONALD B., WILLIAM C. CLARK, DAVID W. CASH, \& NANCY M. DICKSON, eds. 2006. Global Environmental Assessments: Information and Influence. Cambridge, MA: MIT Press.

MORA, C., MYERS, R. A., COLL, M., LIBRALATO, S., PITCHER, T. J., SUMAILA, R. U.,... \& WORM, B. (2009). Management effectiveness of the world's marine fisheries. PLoS Biology, 7(6), e1000131.

PETERSON, M. J. (1992). Whalers, cetologists, environmentalists, and the international management of whaling. International Organization, 46(01), 147-186.

PIELKE, Roger A. (2004). When scientists politicize science: making sense of controversy over The Skeptical Environmentalist. Environmental Science \& Policy, v. 7, n. 5, p. 405-417, 2004.

POLACHECK, T. (2012). Politics and independent scientific advice in RFMO processes: A case study of crossing boundaries. Marine Policy, 36(1), 132-141.

RIDGEWAY, L. (2014). Global level institutions and processes: Frameworks for understanding critical roles and foundations of cooperation and integration. In: 
Garcia, S. M., Rice, J., \& Charles, A. (Eds.). (2014). Governance of Marine Fisheries and Biodiversity Conservation: Interaction and Co-evolution. John Wiley \& Sons.

RODRIK, D. (2001). The global governance of trade: as if development really mattered. Disponível em: < https://www.sss.ias.edu/files/pdfs/Rodrik/Research/globalgovernance-of-trade.pdf $>$. Acesso em: Dezembro, 2017.

ROSENAU, J. (1995). Governance in the Twenty-first Century. Global Governance 1, no. 1. ROSENAU, J.M. \& CZEMPIEL, E. (1992). Governance Without Government: Order and Change in World Politics (Cambridge: Cambridge University Press, 1992).

RUGGIE, J. G. (2014). Global governance and "new governance theory": Lessons from business and human rights. Global Governance, 20(1), 5-17.

STOKKE, O. S. (2001). The interplay of international regimes: Putting effectiveness theory to work. FNI Report 14/2001. Disponível em: < https://www.files.ethz.ch/ isn/100208/01-14-oss.pdf > . Acesso em: Dezembro, 2017.

UNCLOS, 1982. United Nations Convention on the Law of the Sea. Division for Ocean Affairs and the Law of the Sea, UN. Disponível em: www.un.org/Depts/los/ convention_agreements/texts/unclos/unclos_e.pdf > . Acesso em: Abril, 2018.

UNFSA 1995. Agreement for the implementation of the provisions of the United Nations Convention on the Law of the Sea of 10 December 1982, relating to the conservation and management of straddling fish stocks and highly migratory fish stocks. Disponível em: www.un.org/Depts/los/convention_agreements/texts/fish_stocks_agreement/ CONF16 4_37.htm > . Acesso em: Abril, 2018.

YOUDE, J. (2017). Global Health Governance in International Society. Global Governance: A Review of Multilateralism and International Organizations, 23(4), 583-600.

YOUNG, O. R. (1999). The Effectiveness of International Environmental Regimes: Causal Connections and Behavioral Mechanisms. Cambridge, MA: MIT Press.

YOUNG, Oran R. (2017) Governing Complex Systems: Social Capital for the Anthropocene. MIT Press, 2017.

WEBSTER, D. G. (2011). The irony and the exclusivity of Atlantic bluefin tuna management. Marine Policy, 35(2), 249-251.

WILLIAM C., AND GIANDOMENICO MAJONE. (1985). The Critical Appraisal of Scientific Inquiries with Policy Implications. Science, Technology, \& Human Values 10 (3): 6-19. WILLOCK, A. AND LACK, M. (2006) Follow the Leader: Learning from Experience and Best Practice in Regional Fisheries Management Organizations. Disponível em: http://encurtador.com.br/fwNT5 (acessado em Dez 2014).

WWF (2012). Decision on Eastern Atlantic and Mediterranean bluefin tuna follows scientific advice: WWF applauds. Disponível em: < http://mediterranean.panda. org/?206761/Decisions-on-Eastern-Atlantic-and-Mediterranean-bluefin-tuna-followsscientific-advice > . Acesso em: Abril, 2018. 